\title{
The FERRUM Project: Experimental oscillator strengths of the UV 8 multiplet and other UV transitions from the $y^{6} \mathrm{P}$ levels of Fe II
}

\author{
J. C. Pickering ${ }^{1}$, M. P. Donnelly ${ }^{2}$, H. Nilsson ${ }^{3}$, A. Hibbert ${ }^{2}$, and S. Johansson ${ }^{3}$ \\ 1 Physics Department, Blackett Laboratory, Imperial College, London SW7 2BZ, UK \\ 2 Department of Mathematics, Queen's University of Belfast, Northern Ireland \\ 3 Atomic Astrophysics, Lund Observatory, Lund University, PO Box 43, SE-22100 Lund, Sweden
}

Received 13 May 2002 / Accepted 20 September 2002

\begin{abstract}
We report on experimental branching fractions (BFs) for 19 transitions from the three $3 \mathrm{~d}^{5}\left({ }^{6} \mathrm{~S}\right) 4 \mathrm{~s} 4 \mathrm{p}\left({ }^{3} \mathrm{P}\right) y^{6} \mathrm{P}$ levels in Fe II, measured in Fourier transform spectra of a Penning discharge lamp and a hollow cathode lamp. The transition wavelengths span the interval between 1600 and $2850 \AA$. Absolute oscillator strengths ( $f$-values) have been derived by combining the BFs with experimental radiative lifetimes recently reported in the literature. Theoretical $f$-values have been calculated using configuration interaction wavefunctions. The new experimental and theoretical $f$-values are compared with data available in the literature and in databases. The strongest lines measured belong to the UV 8 multiplet of Fe II, which has one ground state transition appearing as a prominent feature at $1608 \AA$ in interstellar spectra. The line is accompanied by a satellite at $1611 \AA$ due to a level mixing, which is discussed in the paper.
\end{abstract}

Key words. atomic data - line: identification - techniques: spectroscopic - stars: abundances - ISM: abundances

\section{Introduction}

Recent years have seen a dramatic increase in the need for accurate and complete atomic data especially in the VUV since the launch of the Hubble Space Telescope (HST) and its High Resolution Spectrograph (GHRS), replaced by the Space Telescope Imaging spectrograph (STIS) in 1999. The effect of the improvements in atomic data for the iron group elements can clearly be seen in the recent analysis of the spectrum of the chemically peculiar star $\chi$ Lupi (Leckrone et al. 1999), but there are still much atomic data that require new or improved measurements.

The UV spectrum of astrophysical plasmas at around $10000 \mathrm{~K}$ is often dominated by Fe II lines, which can serve as a sensitive probe of the plasma conditions. Oscillator strengths ( $f$-values) of weaker lines are needed for determinations of stellar abundances and those of the stronger lines for dilute plasmas. Transitions having large $f$-values but originating from higher energy levels may appear as weak absorption lines in stellar spectra and may be of great use in abundance analyses. The work we report in this paper is part of the collaborative FERRUM Project (Johansson 2001; Johansson et al. 2002 and references therein), in which $f$-values for transitions in the iron group elements are being measured. The aim of the FERRUM Project is to measure $f$-values for transitions in the ultraviolet

Send offprint requests to: J. Pickering, e-mail: j.pickering@ic.ac.uk and optical wavelength regions involving energy levels with an extended span in excitation energy.

In this paper we report on new measurements of branching fractions (BFs) for transitions from the three $3 \mathrm{~d}^{5}\left({ }^{6} \mathrm{~S}\right) 4 \mathrm{~s} 4 \mathrm{p}\left({ }^{3} \mathrm{P}\right)$ $y^{6} \mathrm{P}$ fine structure levels in Fe II. The transitions fall in the UV to VUV spectral range between 1600 and $2850 \AA$ (62 500$35000 \mathrm{~cm}^{-1}$ ). The BFs are measured by high resolution Fourier transform spectrometry and combined with experimental radiative lifetimes reported by $\mathrm{Li}$ et al. (2000) to get absolute oscillator strengths for the lines. We have also calculated these $f$-values using configuration interaction wavefunctions. We compare our new measurements and calculations with previous work.

The first laboratory measurements of $f$-values in the UV8 multiplet $\left(a^{6} \mathrm{D}-y^{6} \mathrm{P}\right)$ of Fe II were reported by Bergeson et al. (1996), who measured the $\lambda 1608$ line using absorption spectroscopy. Mullman et al. (1997) applied the same method and measured $f$-values of six lines in the same multiplet, including the $\lambda 1608$ line. In his extensive work on arc spectra of Fe II Moity (1983) published $f$-values of three lines with $y^{6} \mathrm{P}$ as the upper term. These lines do not belong to the UV 8 multiplet, but to multiplets no. 176 and 190. The earliest measurement of the $f$-value of the $\lambda 1608$ line was made by Shull et al. (1983) quoting an uncertainty of $30 \%$ in a curve-of-growth analysis of interstellar data from the Copernicus and International Ultraviolet Explorer (IUE) satellites. Cardelli \& Savage (1995) discussed the reliability of a number of transition probabilities 
of Fe II lines between 1140 and $2600 \AA$ and determined the $f$-value of the $\lambda 1608$ line using HST/GHRS spectra of $\mu$ Col. They point out the importance of data for UV and VUV transitions of Fe II spanning a wide range of $f$-values. A set of accurate $f$-values allows the iron abundances in interstellar clouds to be studied with a large dynamic range in column density. Ekberg \& Feldman (1993) presented transition probabilities in the 2000-2800 $\AA$ region measured in high resolution spectra of the solar chromosphere including two lines with $y^{6} \mathrm{P}$ as upper term.

There have been a variety of theoretical calculations made for transitions from the three $y^{6} \mathrm{P}$ levels by Nussbaumer et al. (1981), Fawcett (1988), Kurucz (2000), Nahar (1995), Raassen \& Uylings (1998), and Donnelly \& Hibbert (1999).

\section{Intensity measurements}

The iron spectra analysed in this work have been recorded with the VUV Fourier transform (FT) spectrometer at Imperial College, London, UK, and the UV FT spectrometer at Lund Observatory, Lund, Sweden (Thorne et al. 1987). Two different lamps were used as light sources: a Penning discharge (PD) lamp (Heise et al. 1994) and a hollow cathode (HC) lamp.

The spectra recorded with the FT-spectrometer at Imperial College were produced with a PD lamp run with a discharge current of $1.55 \mathrm{~A}$ and with a mixture of neon and argon as the carrier gas at a pressure of $1.7 \times 10^{-3}$ mbar. The entrance window and beamsplitter of this instrument are made of $\mathrm{MgF}_{2}$ giving a detectable signal down to $1350 \AA$ or $74000 \mathrm{~cm}^{-1}$ (Thorne 1996). The optical path in this system was evacuated in order to reduce the $\mathrm{O}_{2}$ absorption at short wavelengths. Three spectral regions were recorded, 24000-47000 $\mathrm{cm}^{-1}$, $31596-61000 \mathrm{~cm}^{-1}$ and $51000-66000 \mathrm{~cm}^{-1}$. The spectra recorded with the Lund instrument were produced by a $\mathrm{HC}$ lamp in the spectral region $25276-50553 \mathrm{~cm}^{-1}$. The $\mathrm{HC}$ was run with 1.3 mbar of neon as the carrier gas and a discharge current of $1.2 \mathrm{~A}$. The recorded interferograms were transformed with a computer code, GREMLIN, developed by J. W. Brault (Brault \& Adams 1989). A Voigt profile was fitted to the lines and the integrated area under the profile was used as the line intensity. In order to obtain relative intensities, the lines were corrected for the instrument response. A deuterium lamp with a $\mathrm{MgF}_{2}$ window calibrated for spectral radiance by the Physikalisch-Technische Bundesanstalt (PTB), Berlin, Germany to within $8 \%$ (two standard deviations $(2 \sigma)$ ) in the range $1660-3580 \AA$, and to within $12 \%$ in the range 1130 $1660 \AA$, was used to determine the instrument response. The light source and the deuterium calibration lamp were placed at similar distances from the FT spectrometer entrance aperture. A mirror was used to switch between the lamps and a lens formed an enlarged image of both sources sufficiently close to the entrance aperture to ensure that they both used the full beamsplitter area. Any potential modulation drifts were allowed for by ensuring that calibration spectra were recorded immediately before and after each set of Fe interferogram coadds. The calibration spectra were compared to check that there had been no change in instrument response during the course of that particular interferogram acquisition. A more detailed description of how the calibration data from PTB was used to determine the instrument response curves is given in Pickering et al. (2001).

The intensities of the strong lines were checked for self absorption, but no indication of this effect was found. Since not all lines could be recorded in one spectrum, several different spectra had to be combined. The intensity scale was transferred from one spectrum to the other by measuring intensity ratios of lines appearing in both spectra in the overlapping regions.

\section{Branching fractions and oscillator strengths}

The BF of a line is defined as the transition probability $\left(A_{\mathrm{ul}}\right)$ of the line divided by the sum of the transition probabilities of all lines from the upper level " $u$ ". Since the relative intensity of a line $\left(I_{\mathrm{ul}}\right)$ is proportional to $A_{\mathrm{ul}}$, the BF can be written as

$$
B F_{\mathrm{ul}}=\frac{I_{\mathrm{ul}}}{\Sigma_{l} I_{\mathrm{ul}}} .
$$

Thus, a correct $B F$ requires that the intensities of all lines from the upper level are measured. However, due to the large number of lines in complex spectra this is in most cases not possible. The weakest lines are not observed and some lines fall outside the recorded wavelength interval. The total intensity of such lines is estimated from theoretical predictions and collected in a residual $B F$. Transitions with $B F<0.2 \%$ could not be detected in the recorded iron spectra, and we used the calculations by Raassen \& Uylings (1998) to estimate the residual. As can be seen in Table 4 the residual is small, about $1 \%$, for all three $y^{6} \mathrm{P}$ levels.

The BFs are converted to absolute transition probabilities $A_{\mathrm{ul}}$ by including the radiative lifetime $\left(\tau_{\mathrm{u}}\right)$ of the upper level according to

$A_{\mathrm{ul}}=\frac{B F_{\mathrm{ul}}}{\tau_{\mathrm{u}}}$.

The lifetimes have recently been measured by Li et al. (2000) using the laser induced fluorescence technique. The $f$-value can be derived from $A_{\mathrm{ul}}$ through

$f=1.499 \times 10^{-16} \frac{g_{\mathrm{u}}}{g_{\mathrm{l}}} \lambda^{2} A_{\mathrm{ul}}$

where $g_{\mathrm{u}}$ and $g_{1}$ are the statistical weights for the upper and lower level, respectively, $\lambda$ is the wavelength in $\AA$, and $A_{\mathrm{ul}}$ is in $\mathrm{s}^{-1}$.

The uncertainties of the $f$-values given in Table 4 are determined according to a recipe suggested by Sikström et al. (2002), and they include the uncertainty of the intensity measurements, the uncertainty arising when combining different spectral regions, the uncertainty in the intensity calibration of the spectra and the uncertainty in the lifetime measurements. 
Table 1. Optimisation processes for the radial functions.

\begin{tabular}{clc}
\hline \hline Orbital & Configurations included & Symmetry \\
\hline $4 \mathrm{p}$ & $3 \mathrm{~d}^{5} 4 \mathrm{~s} 4 \mathrm{p}$ & $y^{6} \mathrm{P}^{\mathrm{o}}$ \\
$5 \mathrm{p}$ & $3 \mathrm{~d}^{6} 4 \mathrm{p}, 3 \mathrm{~d}^{6} 5 \mathrm{p}$ & $z^{6} \mathrm{P}^{\mathrm{o}}$ \\
$6 \mathrm{p}$ & $3 \mathrm{~d}^{5} m \mathrm{~s} n \mathrm{p} ; m, n=4,5,6$ & $y^{6} \mathrm{P}^{\mathrm{o}}$ \\
$5 \mathrm{~s}$ & $3 \mathrm{~d}^{5} 4 \mathrm{~s} 4 \mathrm{p}, 3 \mathrm{~d}^{5} 5 \mathrm{~s} 4 \mathrm{p}$ & $z^{6} \mathrm{P}^{\mathrm{o}}$ \\
$6 \mathrm{~s}$ & $3 \mathrm{~d}^{5} m \mathrm{~s} n \mathrm{p} ; m, n=4,5,6$ & $y^{6} \mathrm{P}^{\mathrm{o}}$ \\
$4 \mathrm{~d}$ & $3 \mathrm{~d}^{6} 4 \mathrm{p}, 3 \mathrm{~d}^{5} 4 \mathrm{~s} 4 \mathrm{p}, 3 \mathrm{~d}^{4} 4 \mathrm{~s} 4 \mathrm{p} 4 \mathrm{~d}$ & $y^{6} \mathrm{P}^{\mathrm{o}}$ \\
$5 \mathrm{~d}$ & $3 \mathrm{~d}^{6} 4 \mathrm{p}, 3 \mathrm{~d}^{5} 4 \mathrm{~s} 4 \mathrm{p}, 3 \mathrm{~d}^{5} 4 \mathrm{p} 4 \mathrm{~d}, 3 \mathrm{~d}^{5} 4 \mathrm{p} 5 \mathrm{~d}$ & $z^{6} \mathrm{P}^{\mathrm{o}}$ \\
$4 \mathrm{f}$ & $3 \mathrm{~d}^{6} 4 \mathrm{~s}, 3 \mathrm{~d}^{5} 4 \mathrm{p} 4 \mathrm{f}$ & $a^{6} \mathrm{D}$ \\
\hline
\end{tabular}

\section{Theoretical calculations of oscillator strengths and transition probabilities}

The oscillator strengths and transition probabilities calculated for the present work were obtained using configuration interaction (CI) wave functions of the form:

$\Psi(J)=\sum_{i=1}^{M} a_{i} \Phi_{i}\left(\alpha_{i} L_{i} S_{i} J\right)$

generated by the code CIV3 (Hibbert 1975; Hibbert et al. 1991). For a specific choice of configuration state functions (CSFs) $\left\{\Phi_{i}\right\}$, where the angular momentum coupling scheme is defined by $\left\{\alpha_{i}\right\}$, the variationally optimal expansion coefficients $\left\{a_{i}\right\}$ are the eigenvector components of the diagonalized Hamiltonian whose typical element is $H_{i j}=\left\langle\Phi_{i}|H| \Phi_{j}\right\rangle$. The corresponding eigenvalue is an upper bound to the energy of the particular state. In particular, if the eigenvalues $\left\{E_{i}\right\}$ are ordered so that $E_{1}<E_{2}<\cdots$, then

$E_{i} \geq E_{i}^{\text {exact }}$

is a consequence of the Hylleraas-Undheim theorem (Hylleraas \& Undheim 1930).

The CSFs of all states are constructed from a common set of one-electron orbitals of the form

$u=\frac{1}{r} P_{n l}(r) Y_{l}^{m_{l}}(\theta, \phi) \chi_{m_{\mathrm{s}}}(\sigma)$

whose angular momenta are coupled to give a total $L_{i} S_{i}$. This may vary from CSF to CSF but their resultant $J$ is common to all CSFs in Eq. (4). The radial functions in Eq. (6) are expressed in analytic form as a linear combination of normalized Slatertype orbitals (STOs):

$P_{n l}(r)=\sum_{j=1}^{k} c_{j n l} \chi_{j n l}(r)$

where the STOs take the form

$\chi_{j n l}(r)=\left[\frac{\left(2 \zeta_{j n l}\right)^{2 I_{j n l}+1}}{\left(2 I_{j n l}\right) !}\right]^{1 / 2} r^{I_{j n l}} \exp \left(-\zeta_{j n l} r\right)$.

Equation (5) constitute a set of variational principles allowing the optimization of the radial function parameters on one or more of the energy eigenvalues of the Hamiltonian matrix. The
Table 2. Optimised radial function parameters.

\begin{tabular}{|c|c|c|c|}
\hline$n l$ & $c_{j n l}$ & $I_{j n l}$ & $\xi_{j n l}$ \\
\hline \multirow[t]{5}{*}{$5 \mathrm{~s}$} & 0.07552 & 1 & 19.02548 \\
\hline & -0.27401 & 2 & 9.35791 \\
\hline & 0.57776 & 3 & 5.07754 \\
\hline & -1.03497 & 4 & 2.43824 \\
\hline & 0.97510 & 5 & 1.29982 \\
\hline \multirow[t]{6}{*}{$6 s$} & 0.06441 & 1 & 18.38583 \\
\hline & -0.16284 & 2 & 11.31165 \\
\hline & 4.62987 & 3 & 2.03315 \\
\hline & -10.89075 & 4 & 2.13989 \\
\hline & 7.93749 & 5 & 2.07416 \\
\hline & -1.98787 & 6 & 1.49966 \\
\hline \multirow[t]{2}{*}{$4 d$} & 0.63694 & 3 & 5.15025 \\
\hline & -0.92313 & 4 & 1.90298 \\
\hline \multirow[t]{3}{*}{$5 d$} & 0.44144 & 3 & 4.73722 \\
\hline & -0.77547 & 4 & 2.61070 \\
\hline & 1.04299 & 5 & 1.28493 \\
\hline \multirow[t]{3}{*}{$4 p$} & 5.70277 & 2 & 1.44819 \\
\hline & -1.88413 & 3 & 4.04161 \\
\hline & -3.85906 & 4 & 2.46303 \\
\hline \multirow[t]{4}{*}{$5 p$} & 0.67165 & 2 & 2.83628 \\
\hline & -0.78204 & 3 & 4.21964 \\
\hline & 5.72815 & 4 & 0.85910 \\
\hline & -6.48385 & 5 & 1.00256 \\
\hline \multirow[t]{5}{*}{$6 p$} & 0.39729 & 2 & 14.25059 \\
\hline & -0.62942 & 3 & 6.78521 \\
\hline & 8.20689 & 4 & 1.38066 \\
\hline & -18.08141 & 5 & 1.37593 \\
\hline & 10.49901 & 6 & 1.42470 \\
\hline $4 \mathrm{f}$ & 1.00000 & 4 & 2.44380 \\
\hline
\end{tabular}

Note: The radial functions of $1 \mathrm{~s}, 2 \mathrm{~s}, 2 \mathrm{p}, 3 \mathrm{~s}, 3 \mathrm{p}, 3 \mathrm{~d}, 4 \mathrm{~s}$ are those given in Table 2 of Clementi \& Roetti (1974) for the ground state of $\mathrm{Fe}^{+}$.

integers $\left\{I_{j n l}\right\}$ are kept fixed but the exponents $\left\{\zeta_{j n l}\right\}$ and the coefficients $\left\{c_{j n l}\right\}$ in Eq. (7) may be treated as variational parameters to be optimized subject to the orthonormality conditions:

$\int_{0}^{\infty} P_{n l}(r) P_{n^{\prime} l}(r) \mathrm{d} r=\delta_{n n^{\prime}} ; \quad l<n^{\prime} \leq n$.

The optimization of the radial functions on, for example, the energy of just one state would lead to a serious imbalance in 
Table 3. Configurations used.

\begin{aligned} & \hline \hline$a^{6} D: 3 d^{6} 4 s, 3 d^{6} 5 s \\ &+ 3 d^{5} 4 s 4 d, 3 d^{5} 5 s 4 d \\ &+ 3 d^{5} 4 p 4 f, 3 d^{5} 5 p 4 f, 3 d^{4} 4 s 4 p 4 f \\ &+ 3 d^{5} 4 p^{2}, 3 d^{5} 4 p 5 p, 3 d^{5} 4 p 6 p \\ &+ 3 d^{4} 4 s 4 d^{2} \\ &. y^{6} P^{0}: 3 d^{6} 4 p, 3 d^{6} 5 p, 3 d^{5} 4 s 4 p, 3 d^{5} 4 s 5 p, 3 d^{5} 5 s 4 p, 3 d^{5} 5 s 5 p \\ &+ 3 d^{5} 4 p 4 d, 3 d^{5} 5 p 4 d, 3 d^{4} 4 s 4 p 4 d, 3 d^{5} 4 p 5 d, 3 d^{5} 5 p 5 d \\ &+ 3 d^{5} 4 s 4 f, 3 d^{5} 4 d 4 f \\ &+ 3 d^{5} 4 s 6 p, 3 d^{5} 5 s 6 p, 3 d^{5} 4 d 6 p \\ &+ 3 d^{4} 4 p 4 d^{2}, 3 d^{4} 5 p 4 d^{2}, 3 d^{3} 4 s 4 p 4 d^{2}, 3 d^{3} 4 s 5 p 4 d^{2}, 3 d^{3} 5 s 4 p 4 d^{2} \\ &$. \hline\end{aligned}

Table 4. Experimental BFs and transition probabilities from the $3 d^{5}\left({ }^{6} \mathrm{~S}\right) 4 \mathrm{~s} 4 \mathrm{p}\left({ }^{3} \mathrm{P}\right) y^{6} \mathrm{P}$ term in Fe II. Air wavelengths above $2000 \AA$ A.

\begin{tabular}{|c|c|c|c|c|c|c|c|c|c|}
\hline \multirow{2}{*}{$\begin{array}{l}\text { Upper } \\
\text { Level }\end{array}$} & \multirow{2}{*}{$\begin{array}{l}\text { Lower } \\
\text { Level }\end{array}$} & \multirow{2}{*}{$\begin{array}{l}\lambda^{1} \\
(\AA)\end{array}$} & \multirow{2}{*}{$\begin{array}{c}\sigma \\
\left(\mathrm{cm}^{-1}\right)\end{array}$} & \multirow{2}{*}{$\begin{array}{r}B F \\
\text { Exp. }\end{array}$} & \multirow{2}{*}{$\begin{array}{l}\text { Uncert } \\
(\% \text { in } g f)\end{array}$} & \multirow{2}{*}{$\begin{array}{c}A\left(\mathrm{~s}^{-1}\right) \\
\text { Exp. }\end{array}$} & \multicolumn{3}{|c|}{$\log g f$} \\
\hline & & & & & & & Exp. & Theory $^{2}$ & Theory $^{3}$ \\
\hline$y^{6} \mathrm{P}_{3 / 2}$ & $b^{2} \mathrm{P}_{1 / 2}$ & 2852.864 & 35042.19 & 0.6 & 17 & $1.65 \mathrm{E}+06$ & -2.09 & -3.36 & -2.10 \\
\hline$E=61974.93 \mathrm{~cm}^{-1}$ & $a^{6} \mathrm{~S}_{5 / 2}$ & 2586.060 & 38657.30 & 2.2 & 37 & $5.75 \mathrm{E}+06$ & -1.64 & -1.75 & -2.11 \\
\hline \multirow[t]{5}{*}{$\tau=3.90 \pm 0.2 \mathrm{~ns}$} & $b^{4} \mathrm{P}_{3 / 2}$ & 2489.110 & 40162.88 & 1.0 & 23 & $2.64 \mathrm{E}+06$ & -2.01 & -2.91 & -2.20 \\
\hline & $a^{6} \mathrm{D}_{1 / 2}$ & 1639.401 & 60997.88 & 28.0 & 7 & $7.18 \mathrm{E}+07$ & -0.94 & -0.93 & -0.99 \\
\hline & $a^{6} \mathrm{D}_{3 / 2}$ & 1636.331 & 61112.31 & 39.3 & 7 & $1.01 \mathrm{E}+08$ & -0.79 & -0.78 & -0.84 \\
\hline & $a^{6} \mathrm{D}_{5 / 2}$ & 1631.129 & 61307.24 & 27.3 & 7 & $7.00 \mathrm{E}+07$ & -0.95 & -0.95 & -1.02 \\
\hline & Residual & & & 1.4 & & & & & \\
\hline$y^{6} \mathrm{P}_{5 / 2}$ & $a^{6} \mathrm{~S}_{5 / 2}$ & 2581.111 & 38731.41 & 2.9 & 10 & $7.61 \mathrm{E}+06$ & -1.34 & -1.38 & -1.80 \\
\hline$E=62049.02 \mathrm{~cm}^{-1}$ & $a^{6} \mathrm{D}_{3 / 2}$ & 1634.349 & 61186.42 & 13.0 & 8 & $3.41 \mathrm{E}+07$ & -1.09 & -1.14 & -1.19 \\
\hline \multirow[t]{3}{*}{$\tau=3.80 \pm 0.2 \mathrm{~ns}$} & $a^{6} \mathrm{D}_{5 / 2}$ & 1629.159 & 61381.35 & 35.1 & 7 & $9.22 \mathrm{E}+07$ & -0.66 & -0.66 & -0.70 \\
\hline & $a^{6} \mathrm{D}_{7 / 2}$ & 1621.685 & 61664.25 & 48.9 & 6 & $1.29 \mathrm{E}+08$ & -0.52 & -0.51 & -0.56 \\
\hline & Residual & & & 0.2 & & & & & \\
\hline$y^{6} \mathrm{P}_{7 / 2}$ & $a^{6} \mathrm{~S}_{5 / 2}$ & 2572.968 & 38853.99 & 2.9 & 8 & $7.89 \mathrm{E}+06$ & -1.20 & -1.24 & -1.53 \\
\hline$E=62171.62 \mathrm{~cm}^{-1}$ & $\mathrm{~b}^{4} \mathrm{~F}_{5 / 2}$ & 2548.157 & 39232.27 & 0.3 & 38 & $8.02 \mathrm{E}+05$ & -2.20 & -2.49 & -2.29 \\
\hline \multirow[t]{8}{*}{$\tau=3.65 \pm 0.2 \mathrm{~ns}$} & $b^{4} \mathrm{~F}_{7 / 2}$ & 2539.805 & 39361.27 & 2.1 & 12 & $5.64 \mathrm{E}+06$ & -1.36 & -1.95 & -1.64 \\
\hline & $b^{4} \mathrm{~F}_{9 / 2}$ & 2528.681 & 39534.42 & 0.8 & 34 & $2.27 \mathrm{E}+06$ & -1.76 & -2.74 & -2.33 \\
\hline & $a^{4} \mathrm{H}_{7 / 2}$ & 2470.847 & 40459.72 & 0.2 & 41 & $5.35 \mathrm{E}+05$ & -2.41 & - & -2.49 \\
\hline & $b^{4} \mathrm{P}_{5 / 2}$ & 2418.168 & 41341.06 & 0.7 & 31 & $2.03 \mathrm{E}+06$ & -1.85 & -2.15 & -2.22 \\
\hline & $a^{6} \mathrm{D}_{5 / 2}$ & 1625.912 & 61503.94 & 4.2 & 12 & $1.15 \mathrm{E}+07$ & -1.44 & -1.55 & -1.58 \\
\hline & $a^{6} \mathrm{D}_{7 / 2}$ & 1618.468 & 61786.83 & 19.9 & 7 & $5.46 \mathrm{E}+07$ & -0.77 & -0.76 & -0.81 \\
\hline & $a^{6} \mathrm{D}_{9 / 2}$ & 1608.451 & 62171.62 & 67.8 & 6 & $1.86 \mathrm{E}+08$ & -0.24 & -0.21 & -0.27 \\
\hline & Residual & & & 1.0 & & & & & \\
\hline
\end{tabular}

${ }^{1}$ Air wavelengths above $2000 \AA$.

${ }^{2}$ Calculations, this work.

${ }^{3}$ Calculations Raassen \& Uylings (1998).

the accuracy to which the full set of states under consideration would be represented. Hence different orbitals are optimized on different eigenvalues, so that all the states are of a comparable level of accuracy and different optimal radial dependencies of the $P_{n l}$ in different states may be accounted for. In the present work, the $1 \mathrm{~s}, 2 \mathrm{~s}, 2 \mathrm{p}, 3 \mathrm{~s}, 3 \mathrm{p}, 3 \mathrm{~d}, 4 \mathrm{~s}$ functions were taken from the ground state Hartree-Fock functions given by Clementi \& Roetti (1974): hence they are all optimized on the $a^{6} \mathrm{D}$ state, and we used the radial functions previously optimized in our calculations of the $a^{6} \mathrm{D}-y{ }^{6} \mathrm{P}^{\mathrm{o}}$ transitions 


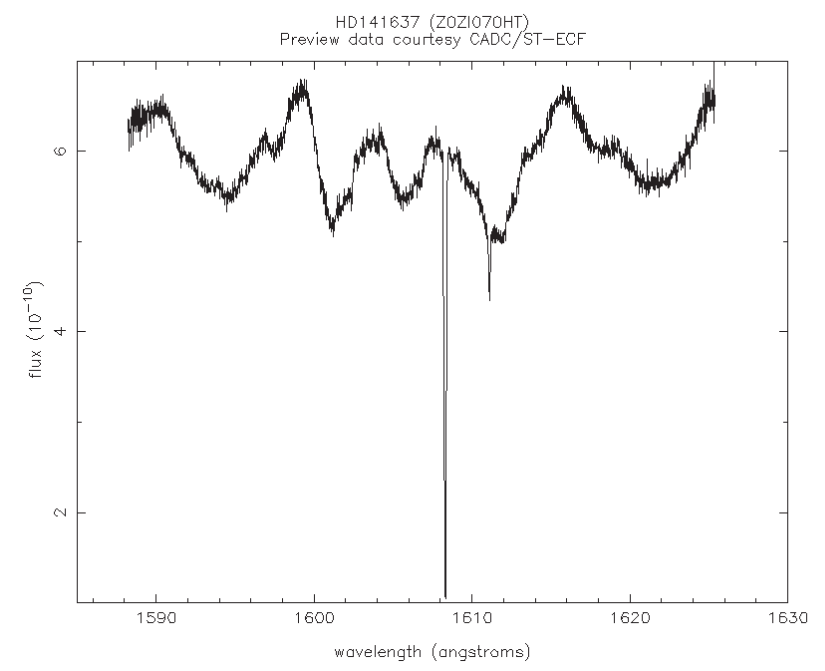

Fig. 1. The GHRS spectrum of the star HD141637 in the vicinity of $1600 \AA$ including the strong (narrow) interstellar Fe II line $\lambda 1608$ $\left(a^{6} \mathrm{D}_{9 / 2}-y^{6} \mathrm{P}_{7 / 2}\right)$, and the satellite line at $1611 \AA\left(a^{6} \mathrm{D}_{9 / 2}-y^{4} \mathrm{~F}_{7 / 2}\right)$, which appears due to level mixing (see text).

(Donnelly \& Hibbert 1998). The additional orbitals consist of $5 \mathrm{~s}, 6 \mathrm{~s}, 4 \mathrm{p}, 5 \mathrm{p}, 6 \mathrm{p}, 4 \mathrm{~d}, 5 \mathrm{~d}, 4 \mathrm{f}$. The method of optimization (involving only the non-relativistic Schrödinger Hamiltonian) is displayed in Table 1 and the radial function parameters are given in Table 2.

The Hamiltonian used to determine the final wave functions consists of the non-relativistic Schrödinger Hamiltonian along with the following relativistic operators associated with the Breit-Pauli approximation: mass-correction, Darwin, spinspin, spin-other-orbit and spin-orbit terms. However, the spinspin-contact and the orbit-orbit terms, which do not contribute directly to fine-structure separations, are not included in the calculations.

When the $L S$ calculation appears adequate, the relativistic effects of the Breit-Pauli Hamiltonian are added, and our $L S J$ calculation is completed. This allows oscillator strengths of individual lines $\left(J \rightarrow J^{\prime}\right)$ to be determined. In this calculation, intercombination lines (where $\Delta S \neq 0$ ) can be determined. They can arise through the mixing of different $L S$ symmetries with a common $J$-value.

Even at this stage the calculated energies will not agree exactly with experiment. This is mainly due to the slow convergence of the summation (Eq. (4)). An extrapolation process known as fine-tuning (Brage \& Hibbert 1989) significantly speeds convergence by making small adjustments to the diagonal elements of the Hamiltonian matrix hence bringing the calculated energies into agreement with experimental energy differences. The results in the following section are those obtained with this 'fine-tuning' process.

This process has proved effective in improving the accuracy of calculated oscillator strengths for ab initio calculation which are already of a high degree of accuracy (Hibbert 1996).

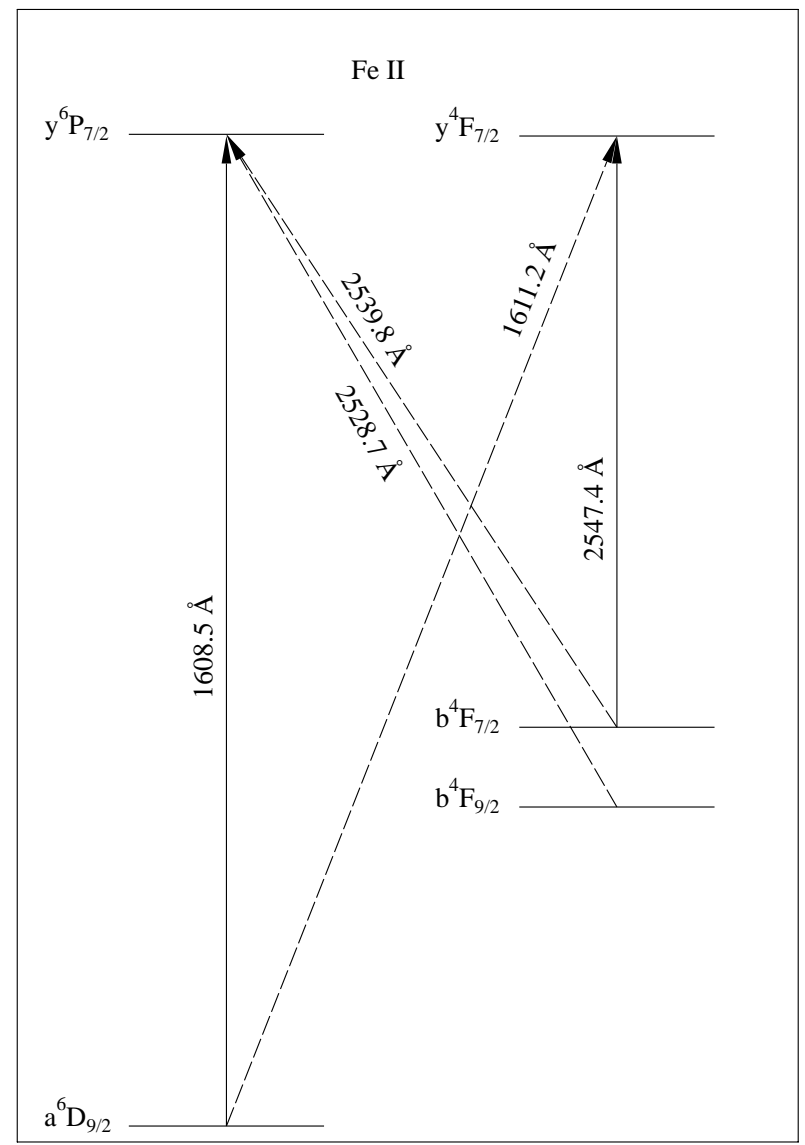

Fig. 2. An illustration of the mixing between the $y^{6} \mathrm{P}_{7 / 2}$ and $y^{4} \mathrm{~F}_{7 / 2}$ levels in Fe II. Three of the resulting intercombination lines appear at $2539.8 \AA, 2528.7 \AA$ and $1611.2 \AA$. The spacing between the $b^{4} \mathrm{~F}$ levels is greatly exaggerated.

\section{Results}

In Table 4 we present the results of our measurements and calculations of the observed lines decaying from the three fine structure levels of $\mathrm{y}^{6} \mathrm{P}$ in Fe II. For each transition we give the wavelength (in air above $2000 \AA$ ), wavenumber and $L S$ notation for the lower energy level. The experimental BFs are tabulated together with transition probabilities, $\log g f$-values (experimental and theoretical) and uncertainties. We also give the $\log g f$-values by Raassen \& Uylings (1998) as these are used for estimating the residual BF for lines that have not been observed.

In general, there is an extremely good agreement between experiment and theory for the sextet transitions, whereas deviations of up to one order of magnitude occur for spin-forbidden (quartet-sextet and even doublet-sextet) transitions, which appear because of level mixing. Since the level mixing is a result of similar energies for levels having the same parity and $J$-value the amount of mixing (and the $\log g f$-values) is strongly sensitive to the accuracy of the calculated level energy. In the next section we show some examples of the effects of level mixing in astrophysical spectra.

In Table 5 we compare the log $g f$-values determined in this work with previous measurements and calculations. Note, that all wavelengths are in vacuum in Table 5, to facilitate the 
Table 5. Comparison between this work and previous work on $\log g f$-values for transitions from $3 \mathrm{~d}^{5}\left({ }^{6} \mathrm{~S}\right) 4 \mathrm{~s} 4 \mathrm{p}\left({ }^{3} \mathrm{P}\right) y^{6} \mathrm{P}$ term in Fe II. All wavelengths are in vacuum.

\begin{tabular}{|c|c|c|c|c|c|c|c|c|c|c|c|c|c|c|}
\hline \multirow{2}{*}{$\begin{array}{l}\lambda_{\mathrm{vac}} \\
(\AA)\end{array}$} & \multirow[t]{2}{*}{ Transition } & \multicolumn{13}{|c|}{$\log g f^{1}$} \\
\hline & & $a$ & $b$ & $c$ & $d$ & $e$ & $f$ & $g$ & $h$ & $i$ & $j$ & $k$ & $l$ & $m$ \\
\hline 1608.451 & $a^{6} \mathrm{D}_{9 / 2}-y^{6} \mathrm{P}_{7 / 2}$ & -0.24 & -0.21 & -0.24 & -0.24 & & -0.27 & -0.129 & -0.016 & -0.37 & -0.049 & -0.21 & & -0.21 \\
\hline 1618.468 & $a^{6} \mathrm{D}_{7 / 2}-y^{6} \mathrm{P}_{7 / 2}$ & -0.77 & -0.76 & -0.75 & & & -0.81 & -0.673 & -0.55 & -0.92 & -0.60 & & & \\
\hline 1621.685 & $a^{6} \mathrm{D}_{7 / 2}-y^{6} \mathrm{P}_{5 / 2}$ & -0.52 & -0.51 & -0.48 & & & -0.56 & -0.443 & -0.31 & -0.67 & -0.34 & & & \\
\hline 1625.912 & $a^{6} \mathrm{D}_{5 / 2}-y^{6} \mathrm{P}_{7 / 2}$ & -1.44 & -1.55 & & & & -1.58 & -1.451 & & & -1.38 & & & \\
\hline 1629.159 & $a^{6} \mathrm{D}_{5 / 2}-y^{6} \mathrm{P}_{5 / 2}$ & -0.66 & -0.66 & -0.77 & & & -0.71 & -0.581 & -0.46 & -0.82 & -0.49 & & & \\
\hline 1631.129 & $a^{6} \mathrm{D}_{5 / 2}-y^{6} \mathrm{P}_{3 / 2}$ & -0.95 & -0.95 & -0.97 & & & -1.02 & -0.889 & -0.76 & -0.90 & -0.78 & & & \\
\hline 1634.349 & $a^{6} \mathrm{D}_{3 / 2}-y^{6} \mathrm{P}_{5 / 2}$ & -1.09 & -1.14 & & & & -1.19 & -1.065 & & & & & & \\
\hline 1636.331 & $a^{6} \mathrm{D}_{3 / 2}-y^{6} \mathrm{P}_{3 / 2}$ & -0.79 & -0.78 & -0.89 & & & -0.84 & -0.713 & -0.58 & -0.95 & -0.61 & & & \\
\hline 1639.401 & $a^{6} \mathrm{D}_{1 / 2}-y^{6} \mathrm{P}_{3 / 2}$ & -0.94 & -0.93 & -1.01 & & & -0.99 & -0.860 & -0.72 & -1.11 & -0.76 & & & \\
\hline 2418.902 & $b^{4} \mathrm{P}_{5 / 2}-y^{6} \mathrm{P}_{7 / 2}$ & -1.85 & -2.15 & & & & -2.22 & -2.706 & & & & & & \\
\hline 2471.594 & $a^{4} \mathrm{H}_{7 / 2}-y^{6} \mathrm{P}_{7 / 2}$ & -2.41 & - & & & & -2.49 & -3.255 & & & & & & \\
\hline 2489.861 & $b^{4} \mathrm{P}_{3 / 2}-y^{6} \mathrm{P}_{3 / 2}$ & -2.01 & -2.91 & & & & -2.20 & -2.484 & & & & & & \\
\hline 2529.441 & $b^{4} \mathrm{~F}_{9 / 2}-y^{6} \mathrm{P}_{7 / 2}$ & -1.76 & -2.74 & & & & -2.33 & -3.980 & & & & & & \\
\hline 2540.568 & $b^{4} \mathrm{~F}_{7 / 2}-y^{6} \mathrm{P}_{7 / 2}$ & -1.36 & -1.95 & & & -1.48 & -1.64 & $-1.720^{2}$ & & & & & $<-1.35$ & \\
\hline 2548.922 & $b^{4} \mathrm{~F}_{5 / 2}-y^{6} \mathrm{P}_{7 / 2}$ & -2.20 & -2.49 & & & & -2.29 & -2.996 & & & & & & \\
\hline 2573.738 & $a^{6} \mathrm{~S}_{5 / 2}-y^{6} \mathrm{P}_{7 / 2}$ & -1.20 & -1.24 & & & -1.29 & -1.53 & $-1.530^{2}$ & & & -1.37 & & $<-1.05$ & \\
\hline 2581.884 & $a^{6} \mathrm{~S}_{5 / 2}-y^{6} \mathrm{P}_{5 / 2}$ & -1.34 & -1.38 & & & -1.46 & -1.80 & $-1.700^{2}$ & & & -1.49 & & & \\
\hline 2586.834 & $a^{6} \mathrm{~S}_{5 / 2}-y^{6} \mathrm{P}_{3 / 2}$ & -1.64 & -1.75 & & & & -2.11 & -2.472 & & & -1.67 & & & \\
\hline 2853.703 & $b^{2} \mathrm{P}_{1 / 2}-y^{6} \mathrm{P}_{3 / 2}$ & -2.09 & -3.36 & & & & -2.10 & -2.337 & & & & & & \\
\hline
\end{tabular}

$\left.{ }^{1} a\right)$ Exp: This work, $b$ ) Theory: This work, $c$ ) Exp: Mullman et al. (1997), d) Exp: Bergeson et al. (1996), e) Exp. Moity (1983), $f$ ) Theory: Raassen \& Uylings (1998), g) Theory: Kurucz (2000), $h$ ) Theory: Nussbaumer et al. (1981), $i$ ) Theory: Facett (1988), $j$ ) Theory: Nahar (1994), k) Astrophysical: Shull et al. (1983), l) Astrophysical: Ekberg \& Feldman (1992), m) Astrophysical: Cardelli \& Savage (1995).

${ }^{2}$ From Fuhr et al. 1988.

comparison with plots of satellite spectra. We give Ritz wavelengths derived from improved energy level values obtained in an ongoing term analysis of Fe II by one of the authors (S.J.). There is a good agreement between our $\log g f$-values and the previous measurements by Bergeson et al. (1996) and Mullman et al. (1997) for the sextet lines, the largest deviation being $0.11 \mathrm{dex}$ ( $25 \%$ in the $f$-value) for the $\lambda 1629$ and $\lambda 1636$ lines. It is especially satisfactory to see the perfect agreement in the log $g f$-value for the important $\lambda 1608$ line not only between the two independent measurements but also with the two independent calculations by Raassen \& Uylings (1998) and by Donnelly \& Hibbert (this work).

\section{Level mixing illustrated by stellar spectra}

The main objective for this work was to measure the $f$-value for the interstellar $\lambda 1608$ line of Fe II and obtain the branching ratio between this fast decay from $y^{6} \mathrm{P}_{7 / 2}$ to the ground state $a^{6} \mathrm{D}_{9 / 2}$ and the decay to the $a^{6} \mathrm{~S}_{5 / 2}$ level at $2573 \AA$. The two lines represent a $3 \mathrm{~d}-4 \mathrm{p}$ and a $4 \mathrm{~s}-4 \mathrm{p}$ transition, respectively, and would look at first glance to be reasonably strong $L S$ transitions. However, the experimental branching ratio is 32 in favour of the $3 \mathrm{~d}-4 \mathrm{p}$ transition, which illustrates the importance of a selection rule for the intermediate $L S$ term arising in the coupling of the $4 \mathrm{~s} 4 \mathrm{p}$ and $4 \mathrm{~s}^{2}$ electrons (Johansson 1978). The $y^{6} \mathrm{P}$ term belongs to the $3 \mathrm{~d}^{5}\left({ }^{6} \mathrm{~S}\right) 4 \mathrm{~s} 4 \mathrm{p}\left({ }^{3} \mathrm{P}\right)$ subconfiguration, with the outer $4 \mathrm{~s}$ and $4 \mathrm{p}$ electrons coupled to a ${ }^{3} \mathrm{P}$ term, whereas the two $4 \mathrm{~s}$ electrons in the $3 \mathrm{~d}^{5}\left({ }^{6} \mathrm{~S}\right) 4 \mathrm{~s}^{2}$ subconfiguration, to which the $\mathrm{a}^{6} \mathrm{~S}$ belongs, are coupled to a ${ }^{1} \mathrm{~S}$ term. Thus, the $4 \mathrm{~s}-4 \mathrm{p}$ transition between $\mathrm{a}^{6} \mathrm{~S}$ and $\mathrm{y}^{6} \mathrm{P}$ disobeys the $L S$ selection rule for the outer $L S$ coupling terms ${ }^{1} \mathrm{~S}$ and ${ }^{3} \mathrm{P}$, which explains its very low branching fraction. The opposite situation is found for the UV multiplet 191, where the $x^{6} \mathrm{P}$ term of the $3 \mathrm{~d}^{5}\left({ }^{6} \mathrm{~S}\right) 4 \mathrm{~s} 4 \mathrm{p}\left({ }^{1} \mathrm{P}\right)$ subconfiguration preferentially decays to $\mathrm{a}^{6} \mathrm{~S}$, obeying the $L S$ selection rule for the outer, intermediate $L S$ terms in a ${ }^{1} \mathrm{~S}-{ }^{1} \mathrm{P}$ transition.

The interstellar $\lambda 1608$ line of Fe II multiplet UV8 appears in a GHRS spectrum of the star HD141637, shown in Fig. 1. Very close on the red side is a much weaker interstellar $\mathrm{Fe}$ II line, which is an appendage to the $\lambda 1608$ line due to level mixing. The $1611 \AA$ A line corresponds to the $L S$-forbidden transition $a^{6} \mathrm{D}_{9 / 2}-y^{4} \mathrm{~F}_{7 / 2}$, also originating from the ground state of Fe II. The upper levels of the two transitions, $y^{6} \mathrm{P}_{7 / 2}$ and $y^{4} \mathrm{~F}_{7 / 2}$, are mixed because they are close in energy, have the same $J$ value and the same parity. The levels are mixed although they belong to different configurations, different spin and $L$ states mainly as a result of the high level density in complex spectra like Fe II. A simple schematic energy level diagram in Fig. 2 illustrates the level mixing. The experimental $\log g f$-value for the $\lambda 1611$ line is -1.86 according to Pickering et al. (2001). 

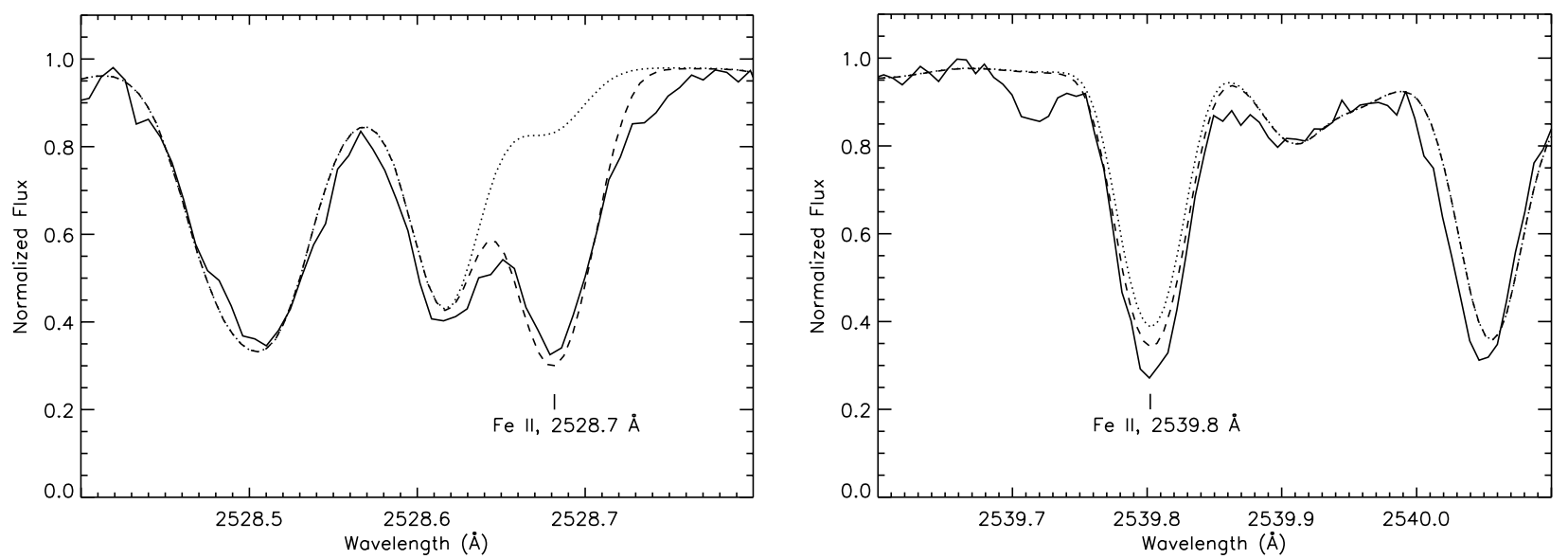

Fig. 3. The two $b^{4} \mathrm{~F}-\mathrm{y}^{6} \mathrm{P}$ Fe II lines at $\lambda \lambda 2528,2539$ in the GHRS spectrum of $\chi$ Lupi. The solid curve represents the recorded spectrum. The dashed curve show the calculated spectra, the with experimental $\log g f$-values from this work and the dotted curve with data from Kurucz (2000).

The level mixing of $y^{6} \mathrm{P}_{7 / 2}$ with $y^{4} \mathrm{~F}_{7 / 2}$ opens a number of additional weak decay channels for each of the levels. The additional transitions from $y^{6} \mathrm{P}_{7 / 2}$ (and a few from the $y^{6} \mathrm{P}_{3 / 2}$ level) can be found in Table 4 . Their branching fractions are typically of the order of a few percent. However, a few percent of a strong line might mean that we see a weak line in a stellar spectrum, which is suitable in abundance work. Since the line is the result of level mixing it might also mean that its log $g f$-value available in theoretical databases is less reliable. In Fig. 3 we have extracted two parts of the GHRS spectrum of the chemically peculiar star $\chi$ Lupi (Leckrone et al. 1999) containing two features, which are identified as two of the Fe II transitions studied in this work. The left panel shows a spectral interval containing a line at $2528.7 \AA$, which is the $b^{4} \mathrm{~F}_{9 / 2}-$ $y^{6} \mathrm{P}_{7 / 2}$ transition shown in Fig. 2 and given in Table 4. The dotted curve represents the calculated spectrum using atomic data from the Kurucz (2000) database which gives a calculated $\log g f$-value $=-3.980$ for $\lambda 2528.7$. The dashed curve is obtained when the Kurucz value is replaced with the new experimental value of -1.76 . As we see in Table 4, other theoretical values for this transition are also too small by up to one order of magnitude. The $b^{4} \mathrm{~F}_{7 / 2}-y^{6} \mathrm{P}_{7 / 2}$ companion to the strong $b^{4} \mathrm{~F}_{7 / 2}-y^{4} \mathrm{~F}_{7 / 2}$ transition at $2547.4 \AA$ appears at $2539.8 \AA$ (see Fig. 2) and shows a large depth in the $\chi$ Lupi spectrum (right panel of Fig. 3). Using the $\log g f$-value for $\lambda 2539.8$ from Fuhr et al. (1988), which is a scaled experimental value from Moity (1983), we obtain the dotted curve, while the new experimental value is used in the dashed curve.

\section{Conclusion}

We present branching fraction measurements for $99 \%$ of the radiative decay from the three $3 \mathrm{~d}^{5}\left({ }^{6} \mathrm{~S}\right) 4 \mathrm{~s} 4 \mathrm{p}\left({ }^{3} \mathrm{P}\right) y^{6} \mathrm{P}$ levels in Fe II and provide accurate oscillator strengths for astrophysical applications. Branching fractions for 19 Fe II lines are measured using spectra recorded with the high resolution FT spectrometers at Imperial College, London, (in the UV-VUV region) and Lund Observatory, Lund University, (in the UV region) having a wide free spectral range, high resolution and linear response. The intensity measurements at Imperial College connect the fundamental $3 d-4 p$ and $4 s-4 p$ transitions in complex spectra, whereas complementary weak lines are measured with the Lund FT spectrometer to obtain as small a residual as possible. FT spectra of Fe II in the range 1520-4160 (24000-66000 $\mathrm{cm}^{-1}$ ) were produced with a PD lamp and a HC lamp. Absolute transition probabilities and $\log g f$-values for the 19 lines are derived by combining the BFs with experimental radiative lifetimes recently reported by Li et al. (2000). The uncertainties in the $f$-values are less than $10 \%$ for strong lines. Our measurements are compared with the experimental work by Bergeson et al. (1996), Mullman et al. (1997) and Moity (1983), and with theoretical calculations by Nussbaumer et al. (1981), Fawcett (1988), Kurucz (2000), Nahar (1995), and Raassen \& Uylings (1998). Our $f$-value (0.058) of the astrophysically important line at $1608 \AA$ coincides with the $f$ values measured by Bergeson et al. (1996) and Mullman et al. (1997) $(f=0.058)$ using an independent method. The theoretical $f$-values of $\lambda 1608$ calculated in this work $(f=0.062)$ and by Raassen \& Uylings (1998) $(f=0.055)$ are also in good agreement with the measurements.

The mixing of the $y^{6} \mathrm{P}$ levels with other levels of the same symmetry, e.g. $y^{4} \mathrm{~F}$, generates faint spectral lines, showing strange $L S$ combinations and having branching fractions smaller than 3\%. However, as illustrated, the lines may show substantial line opacity in high resolution stellar spectra, and could well be used in abundance work. The level mixing also generates a satellite line at 1611 A to the strong interstellar Fe II line at $1608 \AA$.

Acknowledgements. JCP thanks PPARC of the UK for their support of the IC program of measurements of spectra of astrophysical importance. JCP is supported by a Royal Society University Research Fellowship. JCP also thanks Anne Thorne for many helpful discussions. Sadly Richard Learner of our group at IC died recently; JCP is grateful for all his support and contributions to the group's research in atomic spectroscopy for astrophysics over the years. JCP thanks Geoff Cox for his invaluable assistance in the preparatory experimental work using the Penning discharge lamp. The FERRUM Project is supported through a grant (SJ) from the Swedish Natural Science Research Council. MPD and AH thank PPARC (UK) for support under Rolling Grant PPA/G/2000/00024. 


\section{References}

Bergeson, S. D., Mullman, K. L., Wickliffe, M. E., \& Lawler, J. E. 1996, ApJ, 464, 1044.

Brage, T., \& Hibbert, A. 1989, J. Phys. B: At. Mol. Opt. Phys., 22, 713

Brault, J. W., \& Abrams, M. C. 1989, High Resolution Fourier Transform Spectroscopy (OSA Technical Digest Series Vol. 6) (Washington: Optical Society of America), 110

Cardelli, J. A., \& Savage, B. D. 1995, ApJ, 452, 275

Clementi, E., \& Roetti, C. 1974, Atomic Data and Nuclear Data Tables., 14, nos. 3-4, 177

Donnelly, M. P., \& Hibbert, A. 1998, MNRAS, 301, 809

Donnelly, M. P., \& Hibbert, A. 1999, MNRAS, 302, 413

Ekberg, J. O., \& Feldman, U. 1993, ApJS, 86, 611

Fawcett, B. C. 1988, At. Data Nucl. Data Tables, 40, 1

Fuhr, J. R., Martin, G. A., \& Wiese, W. L. 1988, J. Phys. Chem. Ref. Data, 17, Suppl., 4

Heise, C., Hollandt, J., Kling, R., Kock, M., \& Kuhne, M. 1994, Appl. Opt., 33, 5111

Hibbert, A. 1996, Phys. Scr., T65, 104

Hibbert, A. 1975, Comput. Phys. Commun., 9, 141

Hibbert, A., Glass R., \& Froese Fischer, C. 1991, Comput. Phys. Commun., 64, 417

Hylleraas, E. A., \& Undheim, B. 1930, Z. Phys., 65, 759
Johansson, S. 1978, Phys. Scr., 18, 217

Johansson, S. 2001, in press, Proceedings of JD 1, IAU 24th General Assembly, Manchester, UK, August 2000

Johansson, S., Derkatch, A., Donnelly, M., et al. 2002, Phys. Scr., T100, 71

Kurucz, R. L. 2000, http://cfaku5.harvard.edu/

Leckrone, D. S., Proffitt, C. R., Wahlgren, G. M., Johansson, S., \& Brage, T. 1999, AJ, 117, 1454

Li, Z. S., Lundberg, H., Berzinsh, U., Johansson, S., \& Svanberg, S. 2000, J. Phys. B. At. Mol. Opt. Phys., 33, 5593

Moity, J. 1983, A\&AS, 52, 37

Mullman, K. L., Sakai, M., \& Lawler, J. E. 1997, A\&A, 122, 157

Nahar, S. 1995, A\&A, 293, 967

Nussbaumer, H., Pettini, M., \& Storey, P. J. 1981, A\&A, 102, 351

Pickering, J. C., Johansson, S., \& Smith, P. L. 2001, A\&A, 377, 361

Raassen, A. J. J., \& Uylings, P. H. M. 1998, A\&A, 340, 300. The atomic data are available on: ftp://ftp.wins.uva.nl/pub/orth/iron/FeII.E1

Shull, J. M., Van Steenberg, M., \& Seab, C. G. 1983, ApJ, 271, 408

Sikström, C. M., Nilsson, H. Litzén, U. Blom, A., \& Lundberg, H. 2002, J. Quant. Spectrosc. Radiat. Transfer, 74, 355

Thorne, A. P., Harris, C. J., Wynne-Jones, I., Learner, R. C. M., \& Cox, G. 1987, J. Phys. E. Sci. Inst., 20, 54

Thorne, A. P. 1996, Phys. Scr. T Ser., T65, 31 